PROCEEDINGS OF THE

AMERICAN MATHEMATICAL SOCIETY

Volume 112, Number 4, August 1991

\title{
AN EXAMPLE OF A HILBERT TRANSFORM
}

\author{
KRZYSZTOF SAMOTIJ
}

(Communicated by J. Marshall Ash)

\begin{abstract}
We construct a nonnegative integrable function on the real line $\mathbf{R}$ whose Hilbert transform cannot be almost everywhere dominated by the HardyLittlewood maximal function of any finite measure on $\mathbf{R}$.
\end{abstract}

In this paper we construct a nonnegative integrable function on the real line $\mathbf{R}$ whose Hilbert transform cannot be almost everywhere dominated by the (Hardy-Littlewood) maximal function of any finite measure. This answers a question from [1]. The question was motivated by apparent similarities between the magnitude of maximal functions and that of Hilbert transforms as revealed, for example, by the Hardy-Littlewood maximal theorem and Kolmogorov's theorem (see e.g. [4]), Zygmunds theorem [4, Theorem 4.4], Burkholder-GundySilverstein's result [3], and more recent results of Noell and Wolff [5, Proposition 0.6] (reproduced in [1, Theorem 5]) and Bruna and Korenblum [1] (see also [2]). Our present result can be compared with a complementary result of Noell and Wolff [5, p. 40] saying that if $\pi$ is the product measure on the Cantor set then for each finite measure $\mu$ on the real line $\max \{0, M \pi-|H \mu|\} \notin L_{1, \text { loc }}(\mathbf{R})$, where the operators $M$ and $H$ are defined below.

Throughout this paper let a measure be a nonnegative finite Borel measure on the real line $\mathbf{R}$. If $\mu$ is a measure then its Hilbert transform is defined (almost everywhere) as

$$
H \mu(x)=\lim _{\varepsilon \rightarrow 0+} \int_{|x-y| \geq \varepsilon} \frac{d \mu(y)}{x-y}, \quad x \in \mathbf{R} .
$$

Let the maximal function of a measure $\mu$ be the function

$$
M \mu(x)=\sup _{s<x<t} \frac{\mu([s, t])}{t-s}, \quad x \in \mathbf{R} .
$$

If $f$ is a nonnegative integrable function on $\mathbf{R}$ then its Hilbert transform $H f$ and the maximal function $M f$ are defined, respectively, as the Hilbert transform and the maximal function of the measure with the density $f$. In what follows $|A|$ denotes the Lebesgue measure of a set $A, A \subset \mathbf{R}$.

Received by the editors July 10,1989 .

1980 Mathematics Subject Classification (1985 Revision). Primary 42A50.

(c) 1991 American Mathematical Society $0002-9939 / 91 \$ 1.00+\$ .25$ per page 
Our construction makes use of the fact that the maximal function of a measure with the density function equal to the characteristic function of a finite nondegenerate interval multiplied by a constant is bounded by this constant while the Hilbert transform of such a measure has singularities at the endpoints of the interval. This situation will not change much if we replace such a measure by another measure that is close to it. This is expressed in some quantitative terms in the following two lemmas.

Lemma 1. Let a number $\alpha>0$ be arbitrary. Then there are a positive integer $N$ and a positive number $\delta$ such that for each positive $\beta$, each real $a$ and $b, a<$ $b$, each integer $n, n \geq N$, and each measure $\sigma$ of the form $\sigma=\sigma_{1}+\sigma_{2}+\cdots+\sigma_{n}$ with

$$
\sigma_{j}\left(\left[a+\frac{(j-1)(b-a)}{n}, a+\frac{j(b-a)}{n}\right]^{c}\right)=0
$$

and

$$
\sigma_{j}\left(\left[a+\frac{(j-1)(b-a)}{n}, a+\frac{j(b-a)}{n}\right]\right)=\frac{\beta}{n},
$$

$j=1,2, \ldots, n$, we have

$$
\left|\left\{x \in(b+\delta(b-a), b+(b-a)): H \sigma(x) \geq \frac{\alpha \beta}{b-a}\right\}\right| \geq \frac{b-a}{4 e^{\alpha}} .
$$

Proof. Let $\alpha>0$ be arbitrary. It is clear that it is enough to prove the lemma in the case when $a=-1, b=0$, and $\beta=1$. Let $n \geq 2$ and suppose that a measure $\sigma$ satisfies (1) and (2). Then we have

$$
H \sigma_{j}(x) \geq H \chi_{[-1+(j-2) / n,-1+(j-1) / n]}(x), \quad x>-1+j / n, j=1,2, \ldots, n .
$$

Adding these inequalities together we obtain:

$$
H \sigma(x) \geq H \chi_{[-1-1 / n,-1 / n]}(x)=\log \left(\frac{x+1+1 / n}{x+1 / n}\right), \quad x>0 .
$$

Therefore if $n>2 e^{\alpha}$ then $H \sigma(x) \geq \alpha$ provided $0<x \leq\left(2 e^{\alpha}\right)^{-1}$. This completes the proof of the lemma with $\delta=\left(4 e^{\alpha}\right)^{-1}$.

Lemma 2. Let $J_{1}, J_{2}, \ldots, J_{n}$ be a family of open intervals, each of the length $l$, such that the right endpoint of $J_{i}$ is the left endpoint of $J_{i+1}, i=1,2 \ldots, n-1$. Suppose that a measure $\mu$ is concentrated on $\bigcup_{i} J_{i}$ so that $\mu\left(J_{i}\right) \leq B, i=$ $1,2, \ldots, n$, for some constant $B$. Then $M \mu(x) \leq B / l$ whenever $x \geq x_{0}+l$, where $x_{0}$ denotes the right endpoint of $J_{n}$.

Proof. Let $x \geq x_{0}+l$ and let $J$ be an arbitrary finite interval containing $x$. Let $r$ be the number of intervals $J_{i}$ with $J_{i} \cap J \neq \varnothing$. Then $\mu(J)=$ $\sum_{J_{i} \cap J \neq \varnothing} \mu\left(J \cap J_{i}\right) \leq r B$. But since $x \geq x_{0}+l$ we have $r l<|J|$. Therefore $M \mu(x)=\sup _{j \ni x} \mu(J) /|J| \leq B / l$. 
For a nondegenerate finite closed interval $I=[a, b]$, an integer $n, n \geq 2$, and a real number $\theta, 0<\theta<1$, we define

$$
\mathscr{C}(J, n, \theta)=\bigcup_{j=1}^{n}\left[a_{j}, b_{j}\right]
$$

where $a=a_{1}<b_{1}<a_{2}<b_{2}<\cdots<a_{n}<b_{n}=b, b_{j}-a_{j}=\theta|I| / n$, $j=1,2, \ldots, n$ and $a_{2}-b_{1}=a_{3}-b_{2}=\cdots=a_{n}-b_{n-1}$. If $F=\bigcup_{r=1} I_{r}$, where $I_{r}$ 's are pairwise disjoint finite nondegenerate closed intervals then we define

$$
\mathscr{C}(F, n, \theta)=\bigcup_{r=1}^{p} \mathscr{C}\left(I_{r}, n, \theta\right) \text {. }
$$

For given sequences: $\left(n_{k}\right)$ of integers greater than or equal to $2,\left(\theta_{k}\right)$ of real numbers with $0<\theta_{k}<1, k=1,2, \ldots$, we define a decreasing sequence of closed subsets of the line $\mathbf{R}$ inductively as follows:

$$
E_{0}=[0,1], \quad E_{k}=\mathscr{C}\left(E_{k-1}, n_{k}, \theta_{k}\right), \quad k=1,2, \ldots
$$

Each $E_{k}$ is the union of $n_{1} \cdot n_{2} \cdots n_{k}$ disjoint closed intervals which we denote by $I_{j}^{k}, j=1,2, \ldots, n_{1} \cdot n_{2} \cdots \cdots n_{k}$, where $I_{j+1}^{k}$ follows after $I_{j}^{k}$ in the ordering of the real line.

The following lemma is the core of our construction

Lemma 3. Let $\left(n_{k}\right)$ be a sequence of integers greater than or equal to 2. Let $\left(\theta_{k}\right)$ be a sequence of real numbers with $0<\theta_{k}<1, k=1,2, \ldots$. Suppose that $\lim _{k \rightarrow+\infty} n_{k}=+\infty$, and $C=\sup _{k} \theta_{k} \cdot n_{1} \cdot n_{2} \cdots \cdots n_{k}<+\infty$. Then for each positive real $L$ there exists a positive integer $K$ such that if $\nu$ is any measure with $\nu\left(E_{K}^{c}\right)=0$ and $\nu\left(I_{j}^{K}\right)=\left(n_{1} \cdot n_{2} \cdots n_{K}\right)^{-1}, j=1,2, \ldots, n_{1} \cdot n_{2} \cdots n_{K}$, then for each measure $\mu$ with $\mu(\mathbf{R}) \leq L$ we have $|\{x \in(0,2): H \nu(x)>M \mu(x)\}|>0$. Proof. Let us choose $K$ as follows. First, let $\delta$ and $K$ be the numbers from Lemma 1 that correspond to $\alpha=4 L C+2 L+4 C$. Then let $N_{1}$ be an integer greater than or equal to $N$ and such that

$$
n_{k} \geq 3 / \delta+1
$$

and

$$
\theta_{k} \leq 1 / 6
$$

whenever $k \geq N_{1}$. The existence of such an $N_{1}$ follows by the assumption of the lemma. Next, let $N_{2}$ be any integer with

$$
N_{2}>16 e^{\alpha} \text {. }
$$

We will show that $K=N_{1}+N_{2}$ does the job.

For each positive integer $k$ and each $j, j=1,2, \ldots, n_{1} \cdot n_{2} \cdots n_{k}$, let $J_{j}^{k}$ be an interval concentric with $I_{j}^{k}$, chosen so that, for each fixed $k$, the lengths of $J_{j}^{k}$ 's are all equal to the minimum distance between the centers of 


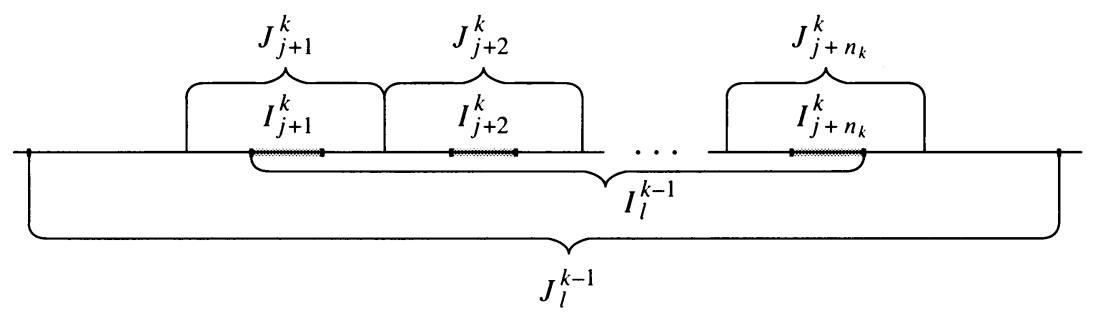

$I_{j}^{k}$ 's. Therefore $J_{j}^{k} \cap J_{i}^{k}=\varnothing$ whenever $j \neq i$. Note that if $I_{j}^{k}$ and $I_{j+1}^{k}$ are subsets of the same $I_{i}^{k-1}$, then the right endpoint of $J_{j}^{k}$ coincides with the left endpoint of $J_{j+1}^{k}$. Note also that

$$
\left|I_{j}^{k}\right|=\frac{\left|E_{k}\right|}{n_{1} \cdots n_{k}}=\frac{\theta_{1} \cdots \theta_{k}}{n_{1} \cdots n_{k}} .
$$

The length of $J_{j}^{k}$ equals $\left(\left|I_{i}^{k-1}\right|-\left|I_{j}^{k}\right|\right) /\left(n_{k}-1\right)$, so, by (6), we have

$$
\left|J_{j}^{k}\right|=\frac{\theta_{1} \cdots \theta_{k-1}}{n_{1} \cdots n_{k}} \frac{n_{k}-\theta_{k}}{n_{k}-1} .
$$

It is easy to see that each $J_{j}^{k+1}$ is contained on some $J_{i}^{k}$.

Let $\mu$ be any measure with $\mu(\mathbf{R}) \leq L$. Let a sequence $(j(k))$ be chosen inductively so that

$$
\mu\left(J_{j(1)}^{1}\right)=\min \left\{\mu\left(J_{i}^{1}\right): i=1,2, \ldots, n_{1}\right\},
$$

and

$$
J_{j(k)}^{k} \subset J_{j(k-1)}^{k-1}, \quad \mu\left(J_{j(k)}^{k}\right)=\min \left\{\mu\left(J_{i}^{k}\right): J_{i}^{k} \subset J_{j(k-1)}^{k-1}\right\}, k \geq 2 .
$$

Since $J_{j}^{1}$ 's are pairwise disjoint we have, by $(8), n_{1} \mu\left(J_{j(1)}^{1}\right) \leq \mu(R) \leq L$. Similarly, (9) implies that $n_{k} \mu\left(J_{j(k)}^{k}\right) \leq \mu\left(J_{j(k-1)}^{k-1}\right), k \geq 2$. Hence the nonnegative sequence $\left(n_{1} \cdots n_{k} \mu\left(J_{j(k)}^{k}\right)\right)$ is nonincreasing and bounded by $L$ from above. Therefore there is a $\tilde{k}, N_{1}<\tilde{k} \leq N_{1}+N_{2}=K$, such that

$$
n_{1} \cdots n_{\tilde{k}-1} \mu\left(J_{j(\tilde{k}-1}^{\tilde{k}-1}\right)-n_{1} \cdots n_{\tilde{k}} \mu\left(J_{j(\tilde{k})}^{\tilde{k}}\right) \leq L / N_{2} .
$$

To simplify the notation we denote $\tilde{I}=[a, b]=I_{j(\tilde{k}-1)}^{\tilde{k}-1}$ and $\tilde{J}=(c, d)=$ $J_{j(\tilde{k}-1)}^{\tilde{k}-1}$. Now we will obtain some estimates of $M \mu(x)$ for $x \in(b+\delta(b-a)$, $b+(b-a))$. First, observe that for such an $x$, by (4), we have

$$
\begin{aligned}
M\left(\left.\mu\right|_{\widetilde{J}^{c}}\right)(x) & \leq \frac{\mu(\mathbf{R})}{\operatorname{dist}\left(x, \widetilde{J}^{c}\right)}=\frac{\mu(\mathbf{R})}{d-x} \\
& \leq \frac{L}{d-[b+(b-a)]}=\frac{L}{|\widetilde{J}| / 2-(3 / 2)|\widetilde{I}|} .
\end{aligned}
$$


Therefore, by (6), (7), (4), and the assumption of the lemma, we have

$$
M\left(\left.\mu\right|_{\widetilde{J}^{c}}\right) \leq \frac{4 L C}{\theta_{1} \cdots \theta_{\tilde{k}-1}}, \quad b+\delta(b-a)<x<b+(b-a) .
$$

In order to estimate $M\left(\left.\mu\right|_{\widetilde{J}}\right)(x)$ denote $\mathscr{J}=\left\{J_{j}^{\tilde{k}}: J_{j}^{\tilde{k}} \subset \widetilde{J}\right\}$ and write $\left.\mu\right|_{\widetilde{J}}=$ $\mu_{1}+\mu_{2}$, where $\mu_{1}$ and $\mu_{2}$ are measures chosen so that $\mu_{1}\left((\cup \mathcal{E})^{c}\right)=0$ and $\mu_{1}(J)=\min \{\mu(J): J \in \mathscr{J}\}=\mu\left(J_{j(\tilde{k})}^{\tilde{k}}\right)$ for each $J \in \mathcal{J}$. Lemma 2 applied to $\mathcal{J}$ and $\mu_{1}$ gives, by (6),

$$
\begin{aligned}
M \mu_{1}(x) & \leq \frac{\mu_{1}\left(J_{j(\tilde{k})}^{k}\right)}{\left|J_{j(\tilde{k})}^{\tilde{k}}\right|}=\frac{n_{1} \cdots n_{\tilde{k}} \mu\left(J_{j(\tilde{k})}^{\tilde{k}}\right)}{n_{1} \cdots n_{\tilde{k}}\left|J_{j(\tilde{k})}^{\tilde{k}}\right|} \\
& \leq \frac{L}{n_{1} \cdots n_{\tilde{k}-1}|\widetilde{I}|}=\frac{L}{\theta_{1} \cdots \theta_{\tilde{k}-1}}
\end{aligned}
$$

if

$$
x \geq \sup (\cup \mathscr{J})+\left|J_{j}^{\tilde{k}}\right|=b+\frac{3}{2}\left|J_{j}^{\tilde{k}}\right|-\frac{1}{2}\left|I_{j}^{\tilde{k}}\right| .
$$

But (6), (7), and (3) imply that the right-hand side of (14) does not exceed $b+\delta|\widetilde{I}|=b+\delta(b-a)$. Hence, by (13) and (14), we have

$$
M \mu_{1}(x) \leq \frac{L}{\theta_{1} \cdots \theta_{\tilde{k}-1}}, \quad x \in(b+\delta(b-a), b+(b-a)) .
$$

To deal with $M \mu_{2}(x)$ let us note that, by (10),

$$
\mu_{2}(\mathbf{R})=\mu(\widetilde{J})-n_{\tilde{k}} \mu\left(J_{j(\tilde{k})}^{\tilde{k}}\right) \leq \frac{L}{N_{2} n_{1} n_{2} \cdots n_{\tilde{k}-1}} .
$$

Therefore by the Hardy-Littlewood maximal theorem, and by (5) and (6), we have

$$
\begin{aligned}
\left|\left\{x \in \mathbf{R}: M \mu_{2}(x) \geq \frac{L}{\theta_{1} \cdots \theta_{\tilde{k}-1}}\right\}\right| & \leq \frac{2 \mu_{2}(\mathbf{R}) \theta_{1} \cdots \theta_{\tilde{k}-1}}{L} \\
& \leq \frac{\theta_{1} \cdots \theta_{\tilde{k}-1}}{8 e^{\alpha} n_{1} \cdots n_{\tilde{k}-1}}=\frac{|\widetilde{I}|}{8 e^{\alpha}} .
\end{aligned}
$$

Since $M \mu \leq M \mu_{1}+M \mu_{2}+M\left(\left.\mu\right|_{\widetilde{J}^{c}}\right)$, combining (12), (15) and (16) we obtain

$$
\left|\left\{x \in(b+\delta(b-a), b+(b-a)): M \mu(x) \geq \frac{4 L C+2 L}{\theta_{1} \cdots \theta_{\tilde{k}-1}}\right\}\right| \leq \frac{|\widetilde{I}|}{8 e^{\alpha}} .
$$

Therefore to complete the proof it is enough to show that

$$
\left|\left\{x \in(b+\delta(b-a), b+(b-a)): H \nu(x) \geq \frac{4 L C+2 L}{\theta_{1} \cdots \theta_{\tilde{k}-1}}\right\}\right|>\frac{|\widetilde{I}|}{8 e^{\alpha}} .
$$


To this end we split $H \nu$ into the sum of $H\left(\left.\nu\right|_{\tilde{I}^{c}}\right)$ and $H\left(\left.\nu\right|_{\tilde{I}}\right)$. The first term can be estimated similarly as $M\left(\left.\mu\right|_{\widetilde{J}}\right)$ was in (11) and (12). Namely, if $b+\delta(b-a)<$ $x<(b-a)$ then

$$
H\left(\left.\nu\right|_{\widetilde{I}^{c}}\right)(x)=H\left(|\nu|_{\widetilde{J}^{c}}\right)(x) \geq \frac{-\nu(\mathbf{R})}{\operatorname{dist}\left(x, \tilde{J}^{c}\right)} \geq \frac{-4 C}{\theta_{1} \cdots \theta_{\tilde{k}-1}} .
$$

To estimate $H\left(\left.\nu\right|_{\tilde{I}}\right)$ note that the measure $\left.\nu\right|_{\tilde{I}}$ satisfies the assumptions of Lemma 1 applied to the interval $\widetilde{I}$ with $\beta=\left(n_{1} \cdots n_{k-1}\right)^{-1}$ and $n=n_{k}$. Hence that lemma together with (6), for $\alpha=4 L C+2 L+4 C$, give

$$
\begin{aligned}
& \left|\left\{x \in(b+\delta(b-a), b+(b-a)): H\left(\left.\nu\right|_{\tilde{I}}\right)(x) \geq \frac{\alpha}{\theta_{1} \cdots \theta_{\tilde{k}-1}}\right\}\right| \\
& \quad \geq \frac{|\widetilde{I}|}{4 e^{\alpha}}>\frac{|\widetilde{I}|}{8 e^{\alpha}} .
\end{aligned}
$$

The inequalities (18) and (19) imply (17).

Lemma 3 implies the following fact which seems interesting enough to call it a theorem.

Theorem 1. Let sequences $\left(n_{k}\right)$ and $\left(\theta_{k}\right)$ satisfy the assumption of Lemma 3 . Let $\pi$ be the product measure on the Cantor-type set $E=\bigcap_{k=1}^{\infty} E_{k}$ associated with these sequences, that is $\pi$ is a probability measure supported on $E$ such that for each $k$ all the components of the set $E_{k}$ have the same $\pi$ measure. Then

$$
|\{x \in(0,2): H \pi(x)>M \mu(x)\}|>0
$$

for each measure $\mu$.

To prove our main theorem let us introduce an auxiliary notation. For each finite closed interval $I$ of the line $\mathbf{R}$ and each real function $g$ defined on $I$ let us set

$$
L_{I}(g)=\inf \{\mu(R): \mu \text { is a measure with } M \mu \geq g \text { a.e. on } I\},
$$

where we assume that $\inf \varnothing=+\infty$. Note that in the definition of $L_{I}(g)$ we can restrict ourselves to measures concentrated on $I$ since for each measure $\mu$ we have $M \mu(x) \leq M\left(\left.\mu\right|_{I}+\mu((-\infty, a)) \delta_{a}+\mu((b,+\infty)) \delta_{b}\right)(x), x \in I$, where $a$ and $b$ are, respectively, the left and the right endpoints of $I$, and $\delta_{a}$ and $\delta_{b}$ are the Dirac deltas.

Lemma 4. For any nondegenerate finite closed interval $I=[a, b]$, and any real $g$ on $I$, and any positive constant $C$ we have

$$
L_{I}(g-C) \geq L_{I}(g)-C|I| .
$$

Proof. Let $\lambda$ be a measure defined by $\lambda(A)=|A \cap I|$, for each Borel subset $A$ of $\mathbf{R}$. For each measure $\mu$ concentrated on $I$, and each $x \in(a, b)$ we have 


$$
\begin{aligned}
M(\mu+C \lambda)(x) & =\sup _{a \leq s<x<t \leq b} \frac{(\mu+C \lambda)([s, t])}{t-s} \\
& =\sup _{a \leq s<x<t \leq b} \frac{\mu([s, t])}{t-s}+C=M \mu(x)+C .
\end{aligned}
$$

Let us suppose that $L_{I}(g-C)<L_{I}(g)-C|I|$. Then there is a measure $\mu$ concentrated on $I$ such that $\mu(\mathbf{R})<L_{I}(g)-C|I|$ and $M \mu \geq g-C$ a.e. on $I$. But, by (20), $M(\mu+C \lambda)=M \mu+C \geq g$ a.e. on $I$, and $(\mu+C \lambda)(\mathbf{R})=$ $\mu(\mathbf{R})+C|I|<L_{I}(g)$. This contradicts the definition of $L_{I}(g)$.

Theorem 2. There is a nonnegative integrable function $g$ on $\mathbf{R}$, vanishing outside $(0,1)$ such that $|\{x \in[0,1]: H g(x)>M \mu(x)\}|>0$ for each measure $\mu$.

Proof. If we fix sequences $\left(n_{k}\right)$ and $\left(\theta_{k}\right)$ satisfying the assumption of Lemma 3 (e.g. $n_{k}=k+1$ and $\theta_{k}=1 /(k+1)$ !) and set $f_{k}=\chi_{E_{k}} /\left|E_{k}\right|, k=1,2, \ldots$, then $\int f_{k}=1$, and, by Lemma $3, \lim _{k \rightarrow+\infty} L_{[0,2]}\left(H f_{k}\right)=+\infty$. It is not hard to see that for any nondegenerate finite closed interval $I=[a, b]$, and arbitrary positive numbers $\varepsilon$ and $L$ there exists a nonnegative integrable function $f$ on $\mathbf{R}$, vanishing outside $I$ such that $\int f=\varepsilon$ and $L_{I}(H f) \geq L$. To construct such a function it is enough to take $f=\varepsilon\left(f_{k} \circ \tau\right) /|I|$ for large enough $k$, where $\tau$ is a linear function with $\tau(a)=0$ and $\tau(b)=2$.

Let $I_{n}=\left[2^{-n}, 3 \cdot 2^{-n-1}\right], n=1,2, \ldots$. Note that $\operatorname{dist}\left(I_{n}, I_{n-1}\right)=2^{-n-1}$, $n=2,3, \ldots$. For each positive integer $n$, let $g_{n}$ be a nonnegative integrable function on $\mathbf{R}$, vanishing outside $I_{n}$ such that $\int g_{n}=2^{-n}$ and

$$
L_{I_{n}}\left(H g_{n}\right) \geq n \text {. }
$$

Let $g=\sum_{n=1}^{\infty} g_{n}$. The function $g$ is nonnegative, vanishes outside $(0,1)$, and $\int g=1$. For each $n \geq 2$ we have

$$
\begin{aligned}
L_{[0,1]}(H g) & \geq L_{I_{n}}\left(H g_{n}+H\left(\sum_{m \neq n} g_{m}\right)\right) \\
& \geq L_{I_{n}}\left(H g_{n}+\inf _{x \in I_{n}} H\left(\sum_{m \neq n} g_{m}\right)\right) .
\end{aligned}
$$

Let $x \in I_{n}$ be arbitrary. Since $g_{m}(y)=0$ whenever $y \geq x$ and $m>n$, we have

$$
H\left(\sum_{m>n} g_{m}\right)(x)=\int_{-\infty}^{x}\left(\sum_{m<n} \frac{g_{m}(y)}{x-y}\right) d y \geq 0 .
$$

On the other hand if $x \in I_{n}$ then

$$
\begin{aligned}
H\left(\sum_{m<n} g_{m}\right)(x) & \geq-\int\left(\sum_{m<n} g_{m}\right) / \operatorname{dist}\left(x, I_{n-1}\right) \\
& \geq-1 / \operatorname{dist}\left(I_{n}, I_{n-1}\right)=-2^{n+1} .
\end{aligned}
$$


Combining (23) and (24) we obtain

$$
\inf _{x \in I_{n}} H\left(\sum_{m \neq n} g_{m}\right)(x) \geq-2^{n+1} \text {. }
$$

Therefore, by (22), Lemma 4, and (21), we have

$$
\begin{aligned}
L_{[0,1]}(H g) & \geq L_{I_{n}}\left(H g_{n}-2^{n+1}\right) \geq L_{I_{n}}\left(H g_{n}\right)-2^{n+1}\left|I_{n}\right| \\
& =L_{I_{n}}\left(H g_{n}\right)-1 \geq n-1 .
\end{aligned}
$$

Since $n$ was arbitrary we have $L_{[0,1]}(H g)=+\infty$, which is all we need to complete the proof.

\section{ACKNOWLEDGMENT}

This work was done while the author was visiting the Department of Mathematics of the State University of New York at Albany. He wishes to thank members of the faculty of this institution, especially Boris Korenblum, for hospitality and valuable discussions.

\section{REFERENCES}

1. J. Bruna and B. Korenblum, On Kolmogorov's theorem, the Hardy-Littlewood maximal function and the radial maximal function, J. Analyse Math. 50 (1988), 225-239.

2. $\ldots$ A note on Calderon-Zygmund singular integral convolution operators, Bull. Amer. Math. Soc. (N.S.) 16 (1987), 271-273.

3. D. L. Burkholder, R. F. Gundy and M. L. Silverstein, A maximal function characterization of the class $H^{p}$, Trans. Amer. Math. Soc. 157 (1971), 137-153.

4. P. L. Duren, Theory of $H^{p}$ spaces, Academic Press, New York and London, 1970.

5. A. V. Noell and T. H. Wolff, On peak sets for Lip $\alpha$ classes, J. Funct. Anal. 86 (1989), 136-179.

Instytut Matematyki, Politechnika Wrociawska, Wybrzeże Wyspiańskiego 27, 50-370 Wroclaw, Poland 